\title{
Partial Deletion of the NR5A1 (SF1) Gene Detected by Synthetic Probe MLPA in a Patient with XY Gonadal Disorder of Sex Development
}

\author{
M. Barbaro ${ }^{a, b} \quad$ M. Cools ${ }^{c} \quad$ L.H.J. Looijengad ${ }^{d}$ S.L.S. Drop ${ }^{e} \quad$ A. Wedella, b \\ ${ }^{a}$ Department of Molecular Medicine and Surgery, Karolinska Institutet, and ${ }^{\mathrm{b} C e n t e r}$ for Inherited Metabolic \\ Diseases (CMMS), Karolinska University Hospital, Stockholm, Sweden; ' Division of Pediatric Endocrinology, \\ Department of Pediatrics, University Hospital Ghent, Ghent, Belgium; ${ }^{d}$ Department of Pathology, \\ Josephine Nefkens Institute, Daniel Den Hoed Cancer Center and 'Division of Pediatric Endocrinology, \\ Department of Pediatrics, Sophia Children's Hospital, Erasmus MC - University Medical Center Rotterdam, \\ Rotterdam, The Netherlands
}

\section{Key Words}

Disorders of sex development - MLPA - NR5A1 •

Steroidogenic factor 1

\begin{abstract}
Steroidogenic factor 1 (SF1, officially NR5A1) is a nuclear receptor involved in adrenal and gonadal development. NR5A1 mutations have been identified in patients with various forms of $46, X Y$ disorders of sex development (DSD), including complete gonadal dysgenesis with or without adrenal insufficiency, mild testicular dysgenesis with ambiguous external genitalia or female external genitalia with clitoromegaly, and penoscrotal hypospadias. We developed a synthetic probe set for MLPA analysis of the NR5A1 gene covering its 7 exons and analyzed 20 patients with 46,XY gonadal DSD in whom analyses failed to identify a genetic cause. We identified a partial NR5A1 deletion affecting exons 2 and 3, leading to NR5A1 haploinsufficiency in 1 patient presenting with female external genitalia with clitoromegaly, absence of a uterus, and mildly dysgenetic testes. This is the first partial NR5A1 gene deletion identified by MLPA in a patient with
\end{abstract}

$46, X Y$ gonadal DSD. This finding stresses the importance of investigating copy number changes, even at the exon level, in genes involved in gonadal DSD. As NR5A1 mutations can cause a wide spectrum of DSD with relatively high frequen$c y$, the analysis of the NR5A1 gene by MLPA is quite important and should be extended to larger groups of patients.

Copyright $\odot 2011$ S. Karger AG, Basel

Steroidogenic factor 1 (SF1, officially named NR5A1) is a nuclear receptor that was initially identified as a regulator of steroidogenesis [Lala et al., 1992]. In mice and humans its expression is initiated in the urogenital ridge and continues in the adrenal glands, gonads, hypothalamus, and pituitary gland [Ingraham et al., 1994; Hanley et al., 1999]. In the developing gonad, NR5A1, together with other transcription factors (i.e. SRY, DAX1, SOX9, and WT1), is involved in gonad determination and differentiation. In testis Leydig cells, NR5A1 activates the expression of several enzymes involved in steroidogenesis, thus leading to the synthesis of testosterone required for Wolffian duct development and the formation of ex-

\section{KARGER}

Fax +4161306 1234

E-Mail karger@karger.ch

www.karger.com (c) $2011 \mathrm{~S}$. Karger AG, Basel

$1661-5425 / 11 / 0054-0181 \$ 38.00 / 0$

Accessible online at:

www.karger.com/sxd
Michela Barbaro

Karolinska Institutet, Department of Molecular Medicine and Surgery

CMM L8:02, Karolinska University Hospital

SE-17176 Stockholm (Sweden)

Tel. + 4685177 3922, E-Mail Michela.Barbaro@ki.se 
ternal genitalia in a male embryo [Ozisik et al., 2003]. In Sertoli cells, NR5A1 activates the expression of AMH, leading to the regression of Müllerian structures. In females, NR5A1 is expressed in the granulosa and theca cells where it regulates genes required for ovarian steroidogenesis and follicle growth maturation [Lourenco et al., 2009].

$S f 1$ null mice lack both adrenals and gonads [Luo et al., 1994], while mice heterozygous (+/-) for a Sf1 deletion present a milder phenotype with adrenal defects and reduced testicular size [Bland et al., 2004].

NR5A1 mutations in humans were initially identified in patients with 46,XY gonadal dysgenesis (GD) and adrenal insufficiency [Achermann et al., 1999, 2002]. However, after the description of heterozygous mutations in some patients with 46,XY gonadal disorders of sex development (DSD) but without adrenal failure [Correa et al., 2004; Mallet et al., 2004], several mutations have been identified in patients with varying degrees of 46 , XY gonadal DSD and apparently normal adrenal function [Kohler et al., 2007; Lin et al., 2007].

Patients with complete 46,XY GD are usually identified at puberty when they present as girls with primary amenorrhea and a 46,XY karyotype or at birth when a girl is born despite a 46,XY karyotype at prenatal testing. In these patients, the absence of testosterone production during embryonic development due to testis dysgenesis leads to regression of the Wolffian structures and to the development of female external genitalia, while the absent production of AMH allows the development of Müllerian structures (Fallopian tubes and uterus). Complete XY GD can generally be discriminated from an XY defect due to impaired androgen action or synthesis, by high gonadotropin values postpubertally, low testosterone levels, and by the presence of a uterus and other Müllerian structures. However, partial forms of GD presenting with ambiguous external genitalia at birth are not always easy to discriminate from other partial defects in androgen production or response.

The phenotypic presentation of XY patients with NR5A1 mutations has turned out to be quite variable, making the evaluation of $46, \mathrm{XY}$ DSD patients complicated. XY patients with NR5A1 mutations can present not only with female external genitalia due to complete testicular dysgenesis but also with clitoromegaly as the only sign of masculinization despite the presence of only mildly dysgenetic testes, thus showing unexpectedly severe underandrogenization of external genitalia compared to the degree of abnormal testicular differentiation [Lin and Achermann, 2008]. Furthermore, Wolffian as well as
Müllerian structures can be present or absent [Lin and Achermann, 2008]. Other patients present with ambiguous external genitalia, resembling the partial androgen insensitivity syndrome [Coutant et al., 2007]. Lately, NR5A1 mutations have also been described in patients with even milder DSD phenotypes such as severe penoscrotal hypospadias [Kohler et al., 2009] and bilateral anorchia with micropenis [Philibert et al., 2007]. Furthermore, the non-synonymous polymorphism Gly146Ala, that slightly diminishes NR5A1 transactivation activity [WuQiang et al., 2003], has been indicated by association studies as a susceptibility factor for micropenis [Wada et al., 2005] and cryptorchidism [Wada et al., 2006]. Recently, missense mutations altering transcriptional activity have been reported in men with severe spermatogenic failure, representing the mildest form of the phenotype spectrum caused by NR $5 A 1$ mutations [Bashamboo et al., 2010].

In 2009, mutations in NR5A1 have also been shown to be associated with ovarian insufficiency in $46, \mathrm{XX}$ women presenting with primary amenorrhea or premature ovarian failure [Lourenco et al., 2009].

The human NR5A1 (SF1) gene on chromosome 9q33.3 is composed of 7 exons, with exons 2-7 being coding while the first exon is non-coding [Oba et al., 1996]. Considering all phenotypes described, more than 30 NR5A1 mutations have been described so far. The mutation type ranges from missense, nonsense, splicing, small deletions, and insertions to entire gene deletions. No clear genotype-phenotype relationship has been documented. Most patients are heterozygous for a mutation, but some patients carrying homozygous mutations with at least $50 \%$ residual activity have been described [Achermann et al., 2002; Lourenco et al., 2009]. In 46,XY DSD cases, mutations have been de novo or inherited from apparently healthy mothers [Lin et al., 2007]; however, in some families, premature ovarian failure can eventually develop in carrier mothers [Lourenco et al., 2009]. Thus, gene dosage and degree of residual NR5A1 activity are both critical for disease presentation. In addition, the penetrance of a mutation is likely affected by modifier genes and environmental factors, as indicated for example by the different phenotypes described in a pair of siblings [Coutant et al., 2007] and in 2 dizygotic twins with the same NR5A1 mutation [Philibert et al., 2007].

Based on these facts, availability of highly informative mutational screening methods for NR5A1 is important, both from a clinical as well as a biological point of view. We therefore developed a synthetic probe set for MLPA analysis of the NR5A1 gene, covering each of the 7 exons. 
Using this approach, $2046, \mathrm{XY}$ gonadal DSD patients from Sweden, the Netherlands, and Belgium were investigated in whom routine analysis failed to identify a genetic cause.

\section{Patients and Methods}

\section{Patients}

Eleven patients with $46, \mathrm{XY}$ GD in whom routine analysis failed to identify a genetic cause were selected among the 46,XY gonadal DSD patients referred to the Clinical Genetic Laboratory of Karolinska University Hospital Stockholm, Sweden. GD was diagnosed by the presence of female or undervirilized external genitalia, menopausal levels of FSH, and gonad biopsies when available. A group of 9 patients with 46,XY gonadal DSD was selected among the 46,XY DSD patients of the Netherlands and Belgium DSD group. The presence of a uterus was not a selection criterion; sometimes this information has been missing in the first patient referral to the genetic clinic, furthermore some patients with NR5A1 mutations have been reported with an absent uterus.

One of the patients presented with adrenal failure in addition to GD. In this case, point mutations in the CYP11A1 gene were excluded. Point mutations in the SRY and NR5A1 genes were excluded in all 20 patients. Karyotype analysis on blood cells did not detect any mosaicism, however, a mosaicism in the gonadal tissue could not be ruled out in all cases because tissue was not available.

All patients were also analyzed by MLPA with a DSD probe set [Barbaro et al., 2008] designed with the aim to identify entire deletions/duplications of genes involved in DSD, especially XY gonadal DSD, and with a 9p24 probe set [Barbaro et al., 2009] to exclude deletions in this region containing several genes, including the DMRT genes 1,2, and 3. No entire gene deletions or duplications were identified. However, in one patient (patient A) a possible NR5A1 partial deletion was identified (see results section for more details). The study was approved by the regional Ethics Committee at the Karolinska Institutet.

DNA was obtained from blood samples, Epstein Barr Virus (EBV)-immortalized cell lines, or gonadal fibroblasts when available.

Patient A is a girl, born from consanguineous parents, who first came to medical attention at 13 years of age, because of progressive virilization (facial hair growth, deepening of the voice, muscular body) and lack of breast development. At presentation, Tanner stage was M1P3A2, clitoral length was $40 \mathrm{~mm}$, and gender identity was female. Discrete clitoromegaly had been noticed at birth but was judged insufficient to prompt medical investigations. The testosterone level was $12.4 \mathrm{nmol} / \mathrm{l}$ (reference value 0.3-0.9 nmol/l), while LH and FSH levels were $5.3 \mathrm{U} / 1$ and 42 $\mathrm{U} / 1$, respectively ( $\mathrm{LH}$ and FSH reference values $2-8 \mathrm{U} / \mathrm{l}$ ). Cytogenetic investigation revealed a 46,XY karyotype. Gonadectomy was performed at laparoscopy. The left and right gonads were found in the inguinal and abdominal position, respectively. Pathological examination of the gonads revealed bilateral testes with pronounced Leydig cell hyperplasia, normal seminiferous tubules, normal germ cell number with incomplete spermato- genesis in the left (inguinal) gonad, and scarce germ cells in the right (abdominal) gonad. Both gonads showed the presence of carcinoma in situ, the precursor lesion of malignant germ cell tumors [Oosterhuis and Looijenga, 2005]. The uterus was absent. After gonadectomy, hormonal replacement therapy was initiated which induced normal breast development. An ACTH test performed at the age of 15 years revealed a normal cortisol response.

\section{$M L P A$}

A specific synthetic probe set for MLPA analysis of the NR5A1 gene, covering each of the 7 exons (table 1) and additional probes for fine-mapping of the encountered deletion (table 2) were designed according to the recommendations by Stern et al. [2004]. The control probes and the pilot probe used have been previously described [Barbaro et al., 2008]. MLPA reactions were performed using the reagents and the recommendations of the EK1 MLPA reagent kit (MRC-Holland, Amsterdam, The Netherlands), starting from $100 \mathrm{ng}$ of genomic DNA. The PCR products were separated by capillary electrophoresis on an ABI 3100 Genetic Analyzer (Applied Biosystems, Warrington, UK). Trace data were analyzed using the Gene Mapper v3.7 software and exported to an Excel spreadsheet (Microsoft). For each sample, the peak heights were first normalized to the average peak height of the control probes followed by normalization to the average peak height of the control samples included in the run. The sample run was considered acceptable if the ratio for the internal control probe was between 0.8 and 1.2. Threshold values for deletion and duplication were set at 0.75 and 1.25 , respectively.

\section{Results}

MLPA analysis using the DSD kit [Barbaro et al., 2008] in patient A resulted in a ratio value for the probe hybridizing to NR5A1 exon 2 consistent with a heterozygous deletion, while the probe for exon 6 showed a value consistent with 2 copies. No other probes targeting NR5A1 are present in the DSD probe set. To exclude the possibility of a false positive result, MLPA analysis was repeated, and exon 2 of NR5A1 was sequenced again. No mutations or silent variants were identified thus supporting the possibility of a partial gene deletion and pinpointing the necessity to confirm and characterize the deletion. Therefore, we developed the SF1 MLPA probe set.

\section{SF1 Probe Set}

The SF1 probe set was first tested in 10 normal controls and in a sample where the entire NR5A1 gene is deleted. All exon probe pairs showed, after normalization, a standard deviation $<10 \%$, thus proving to give reliable and reproducible results (table 1). Furthermore, we tested a sample where one copy of the NR5A1 gene is entirely deleted, kindly donated by Dr. Ravenswaaij-Arts [van Silf- 
Table 1. SF1 probe set

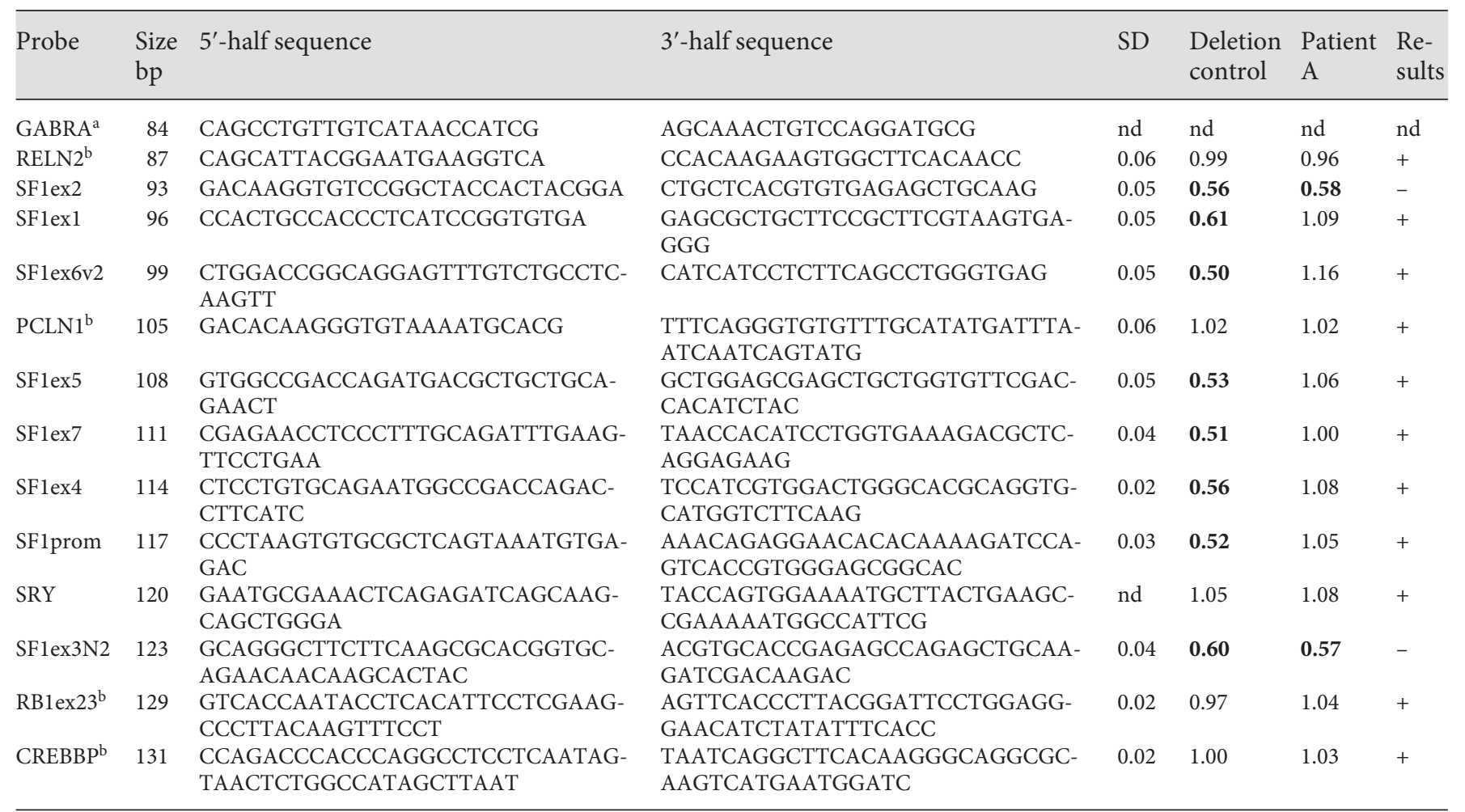

The 5'-half probes are preceded by the universal tag sequence GGGTTCCCTAAGGGTTGGA; the 3'-half probes are followed by the universal tag sequence TCTAGATTGGATCTTGCTGGCAC and are phosphorylated at the $5^{\prime}$-end. Values corresponding to a heterozygous deletion are in bold. Plus and minus signs indicate deleted and non-deleted regions, respectively. nd: Not determined because not relevant. ${ }^{a}$ Pilot probe. ${ }^{b}$ Control probes that hybridize on autosomal chromosomes.

Table 2. Probes used to narrow down the breakpoint in patient $A$

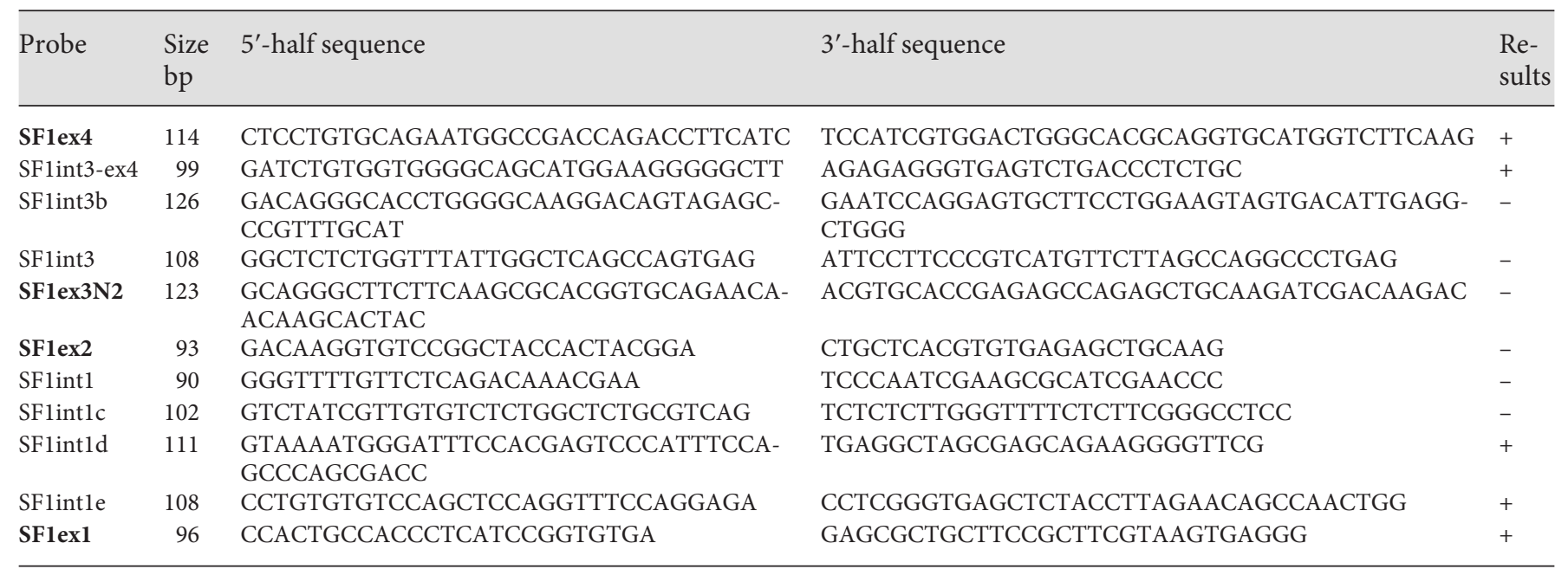

The probes are listed according to their genetic location from the most centromeric to the most telomeric. Probes from the SF1 set that define the initial breakpoint regions are in bold. The 5'-half probes are preceded by the universal tag sequence GGGTTCCCTAAGGGTTGGA; the $3^{\prime}$-half probes are followed by the universal tag sequence TCTAGATTGGATCTTGCTGGCAC and are phosphorylated at the $5^{\prime}$-end. Plus and minus signs indicate non-deleted and deleted regions, respectively. 
Fig. 1. SF1 probe set and SF1 deletion. a Graph showing the results of the MLPA analysis with the SF1 probe set. Samples are normalized using the average of 2 normal controls; for the SRY probe only the male control was used. $\mathrm{Cm}=$ Male control, $\mathrm{Cf}=$ female control, $\mathrm{Cdel}=$ sample with entire $S F 1$ gene deletion, $\mathrm{Pt}=$ patient $\mathrm{A}$. b Representation from the UCSC genome browser (Mar2006, NCBI36, hg18) of the SF1 gene locus on 9q33.3. Probes included in the SF1 set are represented as vertical red lines. c Probes designed to narrow down the breakpoint are represented as vertical green lines. The minimal deletion fragment is represented by a horizontal pink line, with the breakpoint regions delineated by a dotted pink line. The dark green horizontal line indicates the presence of a CpG island. Repeats identified by Repeat Masker are represented by different grey boxes, for the repeats within the breakpoint regions the type is indicated.
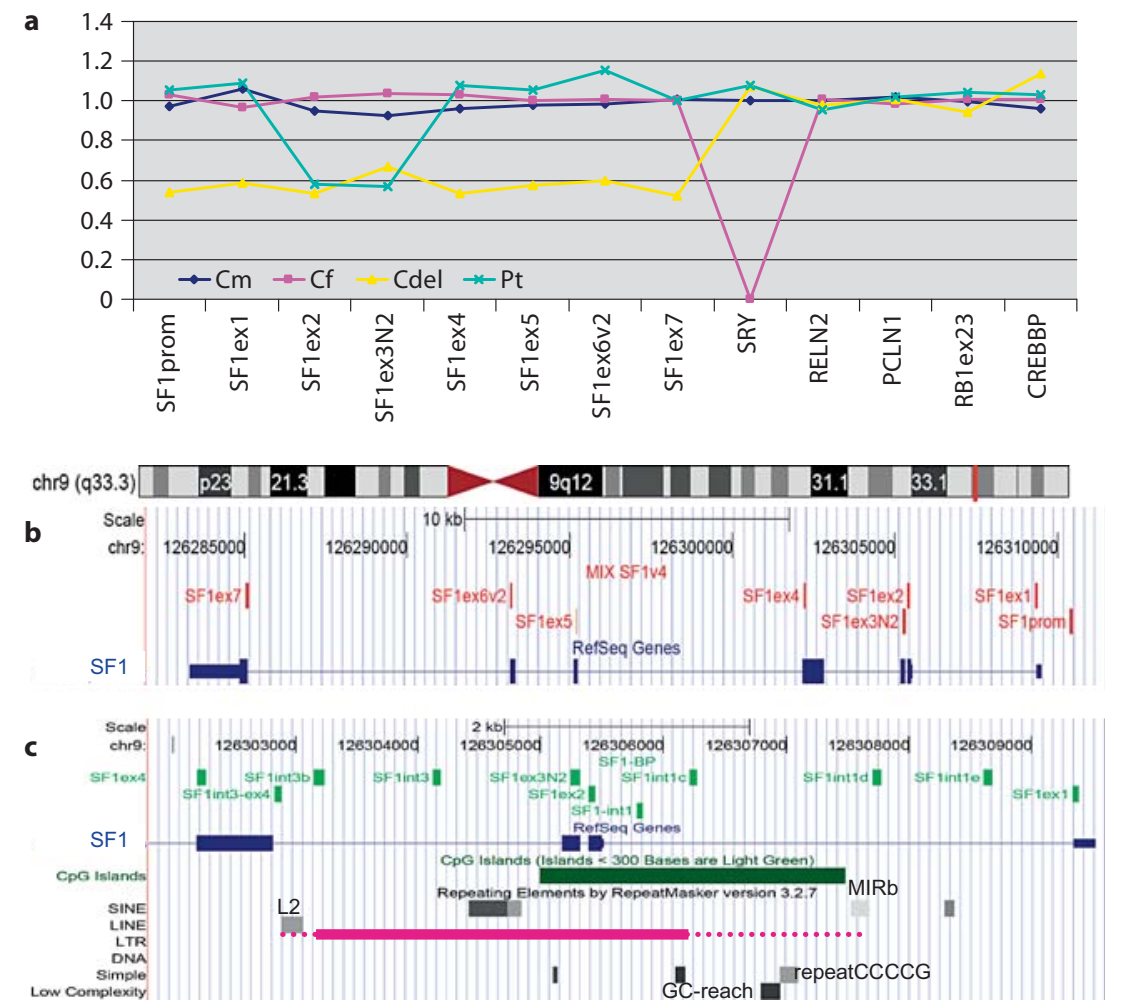

hout et al., 2009]. All probes within NR5A1 had ratio values consistent with a heterozygous deletion, with values ranging from 0.50 to 0.61 (table 1).

\section{Characterization of the Partial NR5A1 Deletion}

In patient $\mathrm{A}$, we could determine that the deletion extended to exon 3 (fig. 1a, b) and almost the entire intron 3. In all the other 19 patients analyzed no copy number changes were identified (data not shown).

Additional synthetic MLPA probes were designed to narrow down the deletion in patient A (table 2). We could establish that the deletion has a size of approximately 3.1$4.8 \mathrm{~kb}$. The telomeric breakpoint lies between the probes SF1intC and SFlint 1d, while the centromeric breakpoint lies between probes SF1int3-ex4 and SF1int3 (table 2, fig. 1c).

The program BLAST (bl2seq; http://blast.ncbi.nlm. nih.gov/Blast.cgi?CMD=Web\&PAGE_TYPE=Blast Home) was used to identify regions of homology that could be involved in mediating the deletion by aligning the 2 breakpoint regions. When the program was optimized for 'somewhat similar sequences (blastn)', a ho- mology of a stretch of $11 \mathrm{bp}$ was identified between the LINE L2 in the centromeric breakpoint region and the SINE MIRb in intron1.

\section{Discussion}

Two entire NR5A1 gene deletions, identified by array$\mathrm{CGH}$, have been reported so far. A $3 \mathrm{Mb}$ heterozygous deletion including NR5A1 and other genes was identified in a 46,XY patient with genitopatellar syndrome, a combination of a XY DSD and nail patella syndrome, the latter most probably caused by haploinsufficiency of the $L M X 1 B$ gene included in the deletion. The XY DSD was characterized by ovotestis, female external genitalia with clitoromegaly, and absence of a uterus [Schlaubitz et al., 2007]. The other NR5A1 deletion had a size of $970 \mathrm{~kb}$, included several genes, and was originated de novo. The $46, \mathrm{XY}$ patient presented with female external genitalia and clitoromegaly, neither uterus nor other Müllerian structures were present. High testosterone levels initially suggested a diagnosis of partial androgen insensitivity 
syndrome [van Silfhout et al., 2009]; no additional syndromic features were described for this patient. Interestingly, although haploinsufficient for the complete NR5A1 gene, both patients presented with some signs of genital virilization (clitoromegaly) and no uterus, indicating a partial gonadal defect.

The identification of such deletions indicates that NR5A1 deletions should be investigated in the evaluation of 46,XY DSD patients. While these 2 deletions could be detected by array-CGH as they were quite large, the detection of deletions affecting only the entire NR5A1 gene, that has a size of approximately $27 \mathrm{~kb}$, by array-CGH is not guaranteed but depends on the practical resolution and the probe distribution of the platform used.

For this reason our group has previously developed a DSD probe set for MLPA analysis of patients with XY DSD in order to identify copy number changes for genes involved in 46,XY DSD. The analysis with this probe set of the Dutch patients detected a deletion affecting the NR5A1 probe in exon 2 but not the NR5A1 probe in exon 6. The possibility of a partial NR5A1 deletion lead us to the development of an additional NR5A1 probe set for MLPA, especially designed to identify NR5A1 single exon deletions/duplications. In addition to the probes targeting each NR5A1 exon, coding and non-coding, we included a probe (SF1prom) located approximately $900 \mathrm{bp}$ upstream of the transcription initiation site in order to detect deletions that affect the promoter region and consequently can impair NR5A1 expression. The basal NR5A1 transcription has been shown to be mainly regulated by the first $110 \mathrm{bp}$ of the proximal promoter that contains several cis-regulatory elements, including a SOX9 binding site (Sox-BS1), an E box, a CCAAT box, and a Sp1 site [Scherrer et al., 2002].

The SF1 probe set was used not only to confirm the partial deletion but also as a diagnostic tool for XY DSD patients where a NR5A1 defect can be suspected.

By designing more MLPA probes, we could determine that patient $A$ carries a deletion affecting only exon 2 and 3 , which are the first 2 coding exons of NR5A1, and almost the entire intron 3 . This is the first description of a partial NR5A1 gene deletion. Within the breakpoint regions there are no highly similar repeats, the only sequence homology identified is a 11-bp sequence shared by the LINE L2 in intron 3 and the SINE MIRb within intron1 (fig. 1C). Despite several attempts, we have not been able to amplify and sequence the deletion junction region. This may be due to the difficulty to perform a long PCR for a region containing a $\mathrm{CpG}$ island. We can, however, exclude a typical non-allelic homologous recombi- nation mechanism as the origin of this deletion and can speculate that the deletion has originated by a double strand breakage followed by a non-homologous end joining mechanism.

No functional NR5A1 can be synthesized from this allele. We actually cannot predict if this allele will even be expressed or not. However, even if mRNA missing exon 2 and 3 is synthesized and initiation of translation occurs at the first in-frame ATG codon (Methionine 88 in exon 4), this would lead to the formation of an N-terminal truncated nuclear receptor missing the DNA binding site which is encoded by exon 2 and 3 .

No further deletions have been identified in the other patients analyzed. For the Swedish patient group this can be due to the patients collected, as all patients presented with complete GD, with female external genitalia (without any sign of virilization) and a uterus. Although some NR5A1 mutations have been identified in patients with complete XY GD, it seems that NR5A1 mutations are more common in patients showing partial/mild XY GD [Lin et al., 2007]. In this respect, the Netherlands and Belgium DSD group of patients selected presented a broader phenotype.

The NR5A1 mutation frequency has been reported as $13 \%$ in 46 , XY patients with GD or impaired androgenization due to impaired Leydig cell function and androgen biosynthesis [Lin et al., 2007], 15\% in patients with severe penoscrotal hypospadias and undescended testis [Kohler et al., 2009], and $33 \%$ in cases of $46, \mathrm{XY}$ females presenting with primary amenorrhea and low testosterone levels [Philibert et al., 2010]. Interestingly, of the 15 patients analyzed by Philibert et al. [2010], 2 patients carried SRY gene mutations that are considered the most common cause of classical XY GD with a frequency of $10-15 \%$, while 5 patients carried mutations in the NR5A1 gene. Two of these 5 patients presented with complete female external genitalia but 3 with clitoromegaly, showing again a higher frequency of NR5A1 mutations when including 46,XY GD patients with partial/mild forms. Thus, NR5A1 mutations are quite frequent in $46, \mathrm{XY}$ DSD. This makes the detection of all types of mutations even more important, as the establishment of a correct genetic diagnosis in XY DSD has several implications for patient management, including sex assignment, treatment choices, gonadal tumor risk evaluation, and accurate genetic counseling to the family.

In conclusion, we describe the first partial NR5A1 gene deletion identified by MLPA in a patient with 46,XY gonadal DSD. This confirms the importance to use a method that can detect copy number changes, even at the 
exon level, in genes involved in gonadal DSD. As NR5A1 mutations can cause a wide spectrum of $46, X Y$ DSD with relatively high frequency and even XX ovarian insufficiency, the analysis of the NR5A1 gene by MLPA is quite important and should be extended to larger groups of patients.

\section{Acknowledgments}

This work was supported by the Stiftelsen Frimurare Barnhuset (to M.B.), the Swedish Research Council (Grant No. 12198), the Novo Nordisk Foundation, Karolinska Institutet, and the Stockholm County Council (to A.W.), and the Flanders Research Foundation (to M.C.).

\section{References}

Achermann JC, Ito M, Ito M, Hindmarsh PC, Jameson JL: A mutation in the gene encoding steroidogenic factor- 1 causes XY sex reversal and adrenal failure in humans. Nat Genet 22: 125-126 (1999).

Achermann JC, Ozisik G, Ito M, Orun UA, Harmanci $\mathrm{K}$, et al: Gonadal determination and adrenal development are regulated by the orphan nuclear receptor steroidogenic factor-1, in a dose-dependent manner. J Clin Endocrinol Metab 87:1829-1833 (2002).

- Barbaro M, Cicognani A, Balsamo A, Lofgren A, Baldazzi L, et al: Gene dosage imbalances in patients with $46, \mathrm{XY}$ gonadal DSD detected by an in-house-designed synthetic probe set for multiplex ligation-dependent probe amplification analysis. Clin Genet 73:453-464 (2008).

- Barbaro M, Balsamo A, Anderlid BM, Myhre AG, Gennari M, et al: Characterization of deletions at $9 p$ affecting the candidate regions for sex reversal and deletion 9p syndrome by MLPA. Eur J Hum Genet 17:14391447 (2009).

- Bashamboo A, Ferraz-de-Souza B, Lourenço D, Lin L, Sebire NJ, et al: Human male infertility associated with mutations in NR5A1 encoding steroidogenic factor 1 . Am J Hum Genet 87:505-512 (2010)

-Bland ML, Fowkes RC, Ingraham HA: Differential requirement for steroidogenic factor-1 gene dosage in adrenal development versus endocrine function. Mol Endocrinol 18:941952 (2004)

-Correa RV, Domenice S, Bingham NC, Billerbeck AE, Rainey WE, et al: A microdeletion in the ligand binding domain of human steroidogenic factor 1 causes $\mathrm{XY}$ sex reversal without adrenal insufficiency. J Clin Endocrinol Metab 89:1767-1772 (2004).

-Coutant R, Mallet D, Lahlou N, Bouhours-Nouet N, Guichet A, et al: Heterozygous mutation of steroidogenic factor-1 in $46, \mathrm{XY}$ subjects may mimic partial androgen insensitivity syndrome. J Clin Endocrinol Metab 92: 2868-2873 (2007)

-Hanley NA, Ball SG, Clement-Jones M, Hagan DM, Strachan T, et al: Expression of steroidogenic factor 1 and Wilms' tumour 1 during early human gonadal development and sex determination. Mech Dev 87:175180 (1999).
Ingraham HA, Lala DS, Ikeda Y, Luo X, Shen $\mathrm{WH}$, et al: The nuclear receptor steroidogenic factor 1 acts at multiple levels of the reproductive axis. Genes Dev 8:2302-2312 (1994).

Kohler B, Lin L, Ferraz-de-Souza B, Wieacker P, Heidemann $P$, et al: Five novel mutations in steroidogenic factor 1 (SF1, NR5A1) in 46,XY patients with severe underandrogenization but without adrenal insufficiency. Hum $\mathrm{Mu}$ tat 29:59-64 (2007)

Kohler B, Lin L, Mazen I, Cetindag C, Biebermann $\mathrm{H}$, et al: The spectrum of phenotypes associated with mutations in steroidogenic factor 1 (SF-1, NR5A1, Ad4BP) includes severe penoscrotal hypospadias in $46, \mathrm{XY}$ males without adrenal insufficiency. Eur J Endocrinol 161:237-242 (2009).

Lala DS, Rice DA, Parker KL: Steroidogenic factor I, a key regulator of steroidogenic enzyme expression, is the mouse homolog of fushi tarazu-factor I. Mol Endocrinol 6:1249-1258 (1992).

Lin L, Achermann JC: Steroidogenic factor-1 (SF-1, Ad4BP, NR5A1) and disorders of testis development. Sex Dev 2:200-209 (2008).

Lin L, Philibert P, Ferraz-de-Souza B, Kelberman D, Homfray T, et al: Heterozygous missense mutations in steroidogenic factor 1 (SF1/Ad4BP, NR5A1) are associated with $46, \mathrm{XY}$ disorders of sex development with normal adrenal function. J Clin Endocrinol Metab 92:991-999 (2007).

-Lourenco D, Brauner R, Lin L, De Perdigo A, Weryha G, et al: Mutations in NR5A1 associated with ovarian insufficiency. N Engl J Med 360:1200-1210 (2009).

Luo X, Ikeda Y, Parker KL: A cell-specific nuclear receptor is essential for adrenal and gonadal development and sexual differentiation. Cell 77:481-490 (1994).

- Mallet D, Bretones P, Michel-Calemard L, Dijoud F, David M, Morel Y: Gonadal dysgenesis without adrenal insufficiency in a 46,XY patient heterozygous for the nonsense C16X mutation: a case of $S F 1$ haploinsufficiency. J Clin Endocrinol Metab 89:4829-4832 (2004).

-Oba K, Yanase T, Nomura M, Morohashi K, Takayanagi R, Nawata H: Structural characterization of human Ad4BP (SF-1) gene. Biochem Biophys Res Commun 226:261-267 (1996).

-Oosterhuis JW, Looijenga LH: Testicular germcell tumours in a broader perspective. Nat Rev Cancer 5:210-222 (2005).
- Ozisik G, Achermann JC, Meeks JJ, Jameson JL: SF1 in the development of the adrenal gland and gonads. Horm Res 59 Suppl 1:94-98 (2003).

Philibert P, Zenaty D, Lin L, Soskin S, Audran F, et al: Mutational analysis of steroidogenic factor 1 (NR5A1) in 24 boys with bilateral anorchia: a french collaborative study. Hum Reprod 22:3255-3261 (2007)

- Philibert P, Leprieur E, Zenaty D, Thibaud E, Polak M, et al: Steroidogenic factor-1 (SF-1) gene mutation as a frequent cause of primary amenorrhea in 46,XY female adolescents with low testosterone concentration. Reprod Biol Endocrinol 8:28 (2010)

Scherrer SP, Rice DA, Heckert LL: Expression of steroidogenic factor 1 in the testis requires an interactive array of elements within its proximal promoter. Biol Reprod 67:15091521 (2002).

- Schlaubitz S, Yatsenko SA, Smith LD, Keller KL, Vissers LE, et al: Ovotestes and XY sex reversal in a female with an interstitial 9q33.3q34.1 deletion encompassing NR5A1 and $L M X 1 B$ causing features of genitopatellar syndrome. Am J Med Genet A 143A:10711081 (2007).

Stern RF, Roberts RG, Mann K, Yau SC, Berg J, Ogilvie CM: Multiplex ligation-dependent probe amplification using a completely synthetic probe set. Biotechniques 37:399-405 (2004).

van Silfhout A, Boot AM, Dijkhuizen T, Hoek A, Nijman R, et al: A unique $970 \mathrm{~kb}$ microdeletion in $9 \mathrm{q} 33.3$, including the NR5A1 gene in a 46,XY female. Eur J Med Genet 52:157-160 (2009).

-Wada Y, Okada M, Hasegawa T, Ogata T: Association of severe micropenis with Gly146Ala polymorphism in the gene for steroidogenic factor-1. Endocr J 52:445-448 (2005).

Wada Y, Okada M, Fukami M, Sasagawa I, Ogata $\mathrm{T}$ : Association of cryptorchidism with Gly146Ala polymorphism in the gene for steroidogenic factor-1. Fertil Steril 85:787-790 (2006).

WuQiang F, Yanase T, Wei L, Oba K, Nomura M, et al: Functional characterization of a new human Ad4BP/Sf-1 variation, G146A. Biochem Biophys Res Commun 311:987-994 (2003). 